\title{
ASYMPTOTIC BOUNDS FOR NORI'S CONNECTIVITY THEOREM
}

\author{
ANIA OTWINOWSKA
}

\begin{abstract}
Let $Y$ be a smooth complex projective variety. I study the cohomology of smooth families of hypersurfaces $\mathcal{X} \rightarrow B$ for $B \subset \mathbb{P H}^{0}(Y, \mathcal{O}(d))$ a codimension $c$ subvariety. I give an asymptotically optimal bound on $c$ and $k$ when $d \rightarrow \infty$ for the space $\mathrm{H}^{k}(Y \times B, \mathcal{X}, \mathbb{Q})$ to vanish, thus extending the validity of Lefschetz Hyperplane section Theorem and Nori's Connectivity Theorem [No. Next, I construct in the limit case explicit families of higher Chow groups whose class does not vanish in $\mathrm{H}^{k}(Y \times B, \mathcal{X}, \mathbb{Q})$. Some of them are indecomposable. This suggests that in the limit case the space $\mathrm{H}^{k}(Y \times B, \mathcal{X}, \mathbb{Q})$ should be spanned by higher Chow groups, containing [No and $\mathrm{O}$ ] as special cases ( $c f$. conjecture 2).
\end{abstract}

\section{INTRODUCTION}

0.1. Nori's connectivity theorem. Let $Y$ be a smooth complex proper algebraic variety of dimension $N+1$ with an ample invertible sheaf $\mathcal{L}$ and $\pi: \mathcal{X} \rightarrow \mathbb{P H}^{0}(Y, \mathcal{L})$ the universal family of hypersurfaces of class $c_{1}(\mathcal{L})$. For any $b \in \mathbb{P H}^{0}(Y, \mathcal{L})$, let $X_{b}=\pi^{-1}(b)$ be the corresponding hypersurface of $Y$.

The Lefschetz hyperplane section theorem asserts that for $k+1 \in\{0, \ldots, N\}$,

$$
\mathrm{H}^{k+1}\left(Y, X_{b}, \mathbb{Z}\right)=0 .
$$

Nori's connectivity theorem extends this result to generically defined cohomology classes of degree all the way up to $2 N=\operatorname{dim}_{\mathbb{R}} X$. More precisely, let $T$ be a smooth variety and $\phi: T \rightarrow \mathbb{P H}^{0}(Y, \mathcal{L})$ a differentiable map. Let $\mathcal{X}_{T}=\mathcal{X} \times_{\mathbb{P H}^{0}(Y, \mathcal{L})} T$ be the universal hypersurface over $T$ and let $\mathcal{Y}_{T}=Y \times T$. By the Leray spectral sequence, the Lefschetz Hyperplane Section Theorem implies that for $k+1 \in\{0, \ldots, N\}$,

$$
\mathrm{H}^{k+1}\left(\mathcal{Y}_{T}, \mathcal{X}_{T}, \mathbb{Z}\right)=0 .
$$

With stronger assumptions one can say more. Assume that $T$ is a smooth algebraic variety and that $\phi: T \rightarrow \mathbb{P H}^{0}(Y, \mathcal{L})$ is a smooth (hence dominant) morphism. Let $\mathcal{O}(1)$ be a fixed very ample invertible sheaf on $Y$ and assume $\mathcal{L}=\mathcal{O}(d)$ for some positive integer $d$.

Theorem (Nori's connectivity theorem $[\mathrm{No}]$ ). For d large enough and $k+1 \in\{0, \ldots, 2 N\}$,

$$
\mathrm{H}^{k+1}\left(\mathcal{Y}_{T}, \mathcal{X}_{T}, \mathbb{Q}\right)=0 \text {. }
$$

Note that Nori's connectivity theorem holds more generally for families of complete intersections in $Y$ (instead of only hypersurfaces). The more general setting is motivated by the construction of algebraic cycles which are homologically but not algebraically equivalent to zero. Since in this paper I do not consider applications to algebraic equivalence, 
I restrict myself to the case of hypersurfaces, which involves all the ideas and simplifies notations.

Despite its topological nature, the proof of Nori's connectivity theorem relies on Hodge theory ( $c f . \mathbb{N o}$, section 1). Namely, let $\Omega_{\mathcal{Y}_{T}, \mathcal{X}_{T}}^{p}$ be the coherent sheaf of $\mathcal{O}_{Y_{T}}$-modules defined by the short exact sequence

$$
0 \rightarrow \Omega_{\mathcal{Y}_{T}, \mathcal{X}_{T}}^{p} \rightarrow \Omega_{\mathcal{Y}_{T}}^{p} \rightarrow j_{T *} \Omega_{\mathcal{X}_{T}}^{p} \rightarrow 0
$$

where $j_{T}: \mathcal{X}_{T} \rightarrow \mathcal{Y}_{T}$ is the closed immersion. Then $\mathrm{H}^{p+q+1}\left(\mathcal{Y}_{T}, \mathcal{X}_{T}, \mathbb{C}\right)=\mathbb{H}^{p+q+1}\left(\mathcal{Y}_{T}, \Omega_{\mathcal{Y}_{T}, \mathcal{X}_{T}}\right)$. Following Nori, let

$$
G^{p} \mathrm{H}^{p+q+1}\left(\mathcal{Y}_{T}, \mathcal{X}_{T}, \mathbb{C}\right)=\operatorname{Im}\left(\mathbb{H}^{p+q+1}\left(\mathcal{Y}_{T}, \Omega_{\mathcal{Y}_{T}, \mathcal{X}_{T}}\right) \rightarrow \mathrm{H}^{p+q+1}\left(\mathcal{Y}_{T}, \mathcal{X}_{T}, \mathbb{C}\right)\right)
$$

denote the Hodge-Grothendick filtration. There is a natural inclusion

$$
F^{p} \mathrm{H}^{p+q+1}\left(\mathcal{Y}_{T}, \mathcal{X}_{T}, \mathbb{C}\right) \subset G^{p} \mathrm{H}^{p+q+1}\left(\mathcal{Y}_{T}, \mathcal{X}_{T}, \mathbb{C}\right)
$$

where $F^{p} \mathrm{H}^{p+q+1}\left(\mathcal{Y}_{T}, \mathcal{X}_{T}, \mathbb{C}\right)$ denotes the Hodge filtration of the mixed Hodge structure on $\mathrm{H}^{p+q+1}\left(\mathcal{Y}_{T}, \mathcal{X}_{T}\right)$. Hence if for some positive integer $k$ one has $\mathrm{H}^{q+1}\left(\mathcal{Y}_{T}, \Omega_{\mathcal{Y}_{T}, \mathcal{X}_{T}}^{p}\right)=0$ for all $p+q+1 \leq k+1$ and $q \leq(k+1) / 2$, then by the Hodge-Fröhlicher spectral sequence $G^{k+1-[(k+1) / 2]} \mathrm{H}^{k+1}\left(\mathcal{Y}_{T}, \mathcal{X}_{T}, \mathbb{C}\right)=0$, hence $F^{k+1-[(k+1) / 2]} \mathrm{H}^{k+1}\left(\mathcal{Y}_{T}, \mathcal{X}_{T}, \mathbb{C}\right)=0$ and a mixed Hodge structures argument implies $\mathrm{H}^{k+1}\left(\mathcal{Y}_{T}, \mathcal{X}_{T}, \mathbb{Q}\right)=0$. Hence the connectivity theorem follows from the following more precise Hogde-theoretical statement

Theorem (Nori). For d large enough, $p+q+1 \leq 2 N$ and $q+1 \leq N$,

$$
\mathrm{H}^{q+1}\left(\mathcal{Y}_{T}, \Omega_{\mathcal{Y}_{T}, \mathcal{X}_{T}}^{p}\right)=0
$$

0.2. Vanishing theorems. The first goal of this paper is to investigate the minimal assumptions for Nori's connectivity theorem to extend. Natural questions are:

- how large does $d$ need to be?

- what happens if the map $\phi: T \rightarrow \mathbb{P H}^{0}(Y, \mathcal{O}(d))$ fails to be dominant, i.e if one considers families of hypersurfaces which are not general, but close to being general?

- can the above assertions be made more precise for a fixed integer $k$ (in Nori's connectivity theorem), and for fixed integers $p$ and $q$ (in the Hodge-theoretical version)?

The first question was studied in Nagel $[\mathrm{N}]$ and Voisin $[\mathrm{V2}$. In this paper I give the following asymptotic answer to the above questions.

Let $T$ be a smooth algebraic variety with an algebraic morphism $\phi: T \rightarrow \mathbb{P H}^{0}(Y, \mathcal{O}(d))$, such that $\mathcal{X}_{T}$ is smooth. I assume that for all $t \in T$ the cokernel of the differential map $d \phi(t): T_{T, t} \rightarrow T_{\mathbb{P H}^{0}(Y, \mathcal{O}(d)), \phi(t)}$ is of dimension at most an integer $c$.

Theorem 1. For all $\varepsilon \in] 0,1[$ and for all $p \in \mathbb{N}$ and for $q+1 \in\{0, \ldots, N\}$, there is an integer $D$ depending only on $\varepsilon, p, Y$ and $\mathcal{O}(1)$, such that for all $d \geq D$,

$$
\mathrm{H}^{q+1}\left(\mathcal{Y}_{T}, \Omega_{\mathcal{Y}_{T}, \mathcal{X}_{T}}^{p}\right)=0
$$


provided that

$$
c \leq(1-\varepsilon) \frac{d^{N-q}}{(N-q) !}
$$

A straightforward adaptation of Nori's argument sketched above shows that in the numerical situation of Theorem $1 G^{p} \mathrm{H}^{p+q+1}\left(\mathcal{Y}_{T}, \mathcal{X}_{T}, \mathbb{C}\right)=0$, hence $F^{p} \mathrm{H}^{p+q+1}\left(\mathcal{Y}_{T}, \mathcal{X}_{T}, \mathbb{C}\right)=$ 0 , and the following topological statement holds.

Corollary . For all $\varepsilon \in] 0,1[$ and $k+1 \in\{0, \ldots, 2 N\}$ there is an integer $D$ depending only on $\varepsilon, Y$ and $\mathcal{O}(1)$ such that for all $d \geq D$,

$$
\mathrm{H}^{k+1}\left(\mathcal{Y}_{T}, \mathcal{X}_{T}, \mathbb{Q}\right)=0
$$

provided that

$$
c \leq(1-\varepsilon) \frac{d^{N-[k / 2]}}{(N-[k / 2]) !} .
$$

The following remarks show that the bounds given by Theorem 1 and the Corollary are close to being asymptotically optimal:

0.2.1. Case $k+1 \in\{0, \ldots, N\}$. By the Lefschetz hyperplane section theorem and the Leray spectral sequence one has $\mathrm{H}^{k+1}\left(\mathcal{Y}_{T}, \mathcal{X}_{T}, \mathbb{Q}\right)=0$ with no assumption on $c$. (similarily, by the holomorphic Leray spectral sequence one has $\mathrm{H}^{q+1}\left(\mathcal{Y}_{T}, \Omega_{\mathcal{Y}_{T}, \mathcal{X}_{T}}^{p}\right)=0$ for $p+q<N$ with no assumption on $c$ ).

0.2.2. Case $k+1 \in\{N+1, \ldots, 2 N\}$. I show in the next section (see Theorem 3 below) that under a weak assumption on $Y$, the bound on $c$ is asymptotically optimal for any couple of fixed integers $p$ and $q$ such that $N / 2 \leq q \leq N-1$ and $p \geq q$. This implies that the bound given by the Corollary is asymptotically optimal.

0.2.3. Case $k+1 \geq 2 N+1$. One has $\mathrm{H}^{2 N+1}\left(\mathcal{Y}_{T}, \mathcal{X}_{T}, \mathbb{Q}\right) \neq 0$ for any positive integer $c$, $d>N$ and $T=\mathcal{X}_{B}$, where $B \subset \mathbb{P H}^{0}(Y, \mathcal{O}(1))$ is any smooth codimension $c$ subvariety contained in the locus of smooth hypersurfaces. Indeed, the condition $d>N$ implies $\mathrm{H}^{0, N}\left(X_{b}, \mathbb{Q}\right) \neq 0$ for all $b \in B$, hence $(c f$. $\mathbb{N o}$, section 0.2$)$ the cohomology class of the diagonal embedding of $\mathcal{X}_{B}$ in $\mathcal{X}_{T}=\mathcal{X}_{B} \times_{B} \mathcal{X}_{B}$ is an element of $\mathrm{H}^{2 N}\left(\mathcal{X}_{T}, \mathbb{Q}\right)$ which does not belong to $j_{T}^{*} \mathrm{H}^{2 N}\left(\mathcal{Y}_{T}, \mathbb{Q}\right)$, hence defines a non-zero element of $\mathrm{H}^{2 N+1}\left(\mathcal{Y}_{T}, \mathcal{X}_{T}, \mathbb{Q}\right)$.

0.3. Some examples of algebraic classes. The aim of this section is to give explicit constructions of algebraic classes in $F^{p} \mathrm{H}^{p+q+1}\left(\mathcal{Y}_{T}, \mathcal{X}_{T}, \mathbb{C}\right)$ for $q+1 \in\{0, \ldots N\}, p \geq q$ and $c$ close to the bound given by theorem 1

0.3.1. The regulator map. For any smooth algebraic variety $X$ and positive integers $p \geq q$ the higher Chow groups $\mathrm{CH}^{p}(X, p-q)$ are defined in $[\mathrm{B}]$. In this paper they are always implicitely assumed to be with $\mathbb{Q}$ coefficients. They come with the Deligne class map

$$
\mathrm{cl}_{p, p-q}(\mathcal{D}): \mathrm{CH}^{p}(X, p-q) \rightarrow \mathrm{H}_{\mathcal{D}}^{p+q}(X, \mathbb{Q}(p)),
$$

where $\mathrm{H}_{\mathcal{D}}^{p+q}(X, \mathbb{Q}(p))$ denotes Deligne cohomology. There is a natural map

$$
r_{p, p-q}: \mathrm{H}_{\mathcal{D}}^{p+q}(X, \mathbb{Q}(p)) \rightarrow F^{p} \mathrm{H}^{p+q}(X, \mathbb{C}),
$$


where $F^{p} \mathrm{H}^{p+q}(X, \mathbb{C})$ denotes the Hodge filtration. Composing $\mathrm{cl}_{p, p-q}(\mathcal{D})$ with $r_{p, p-q}$ one gets the regulator map

$$
\operatorname{reg}_{p, p-q}: \mathrm{CH}^{p}(X, p-q) \rightarrow F^{p} \mathrm{H}^{p+q}(X, \mathbb{C}) .
$$

Note that this map is identically zero for $p>q$ whenever $X$ is proper.

Consider the case $X=\mathcal{X}_{T}$ (note that $\mathcal{X}_{T}$ is not proper). Let

$$
\mathrm{H}^{p+q}\left(\mathcal{X}_{T}, \mathbb{C}\right)_{v}=\operatorname{coker}\left(j_{T}^{*}: \mathrm{H}^{p+q}\left(\mathcal{Y}_{T}, \mathbb{C}\right) \rightarrow \mathrm{H}^{p+q}\left(\mathcal{X}_{T}, \mathbb{C}\right)\right)
$$

denote the vanishing cohomology, let $F^{p} \mathrm{H}^{p+q}\left(\mathcal{X}_{T}, \mathbb{C}\right)_{v}=F^{p} \mathrm{H}^{p+q}\left(\mathcal{X}_{T}, \mathbb{C}\right) \cap \mathrm{H}^{p+q}\left(\mathcal{X}_{T}, \mathbb{C}\right)_{v}$ and let

$$
\overline{\operatorname{reg}}_{p, p-q}: \mathrm{CH}^{p}\left(\mathcal{X}_{T}, p-q\right) \rightarrow F^{p} \mathrm{H}^{p+q}\left(\mathcal{X}_{T}, \mathbb{C}\right)_{v} \subset F^{p} \mathrm{H}^{p+q+1}\left(\mathcal{Y}_{T}, \mathcal{X}_{T}, \mathbb{C}\right)
$$

be the map deduced from $\operatorname{reg}_{p, p-q}$ by projection on $\mathrm{H}^{p+q}\left(\mathcal{X}_{T}, \mathbb{C}\right)_{v}$.

An element of $F^{p} \mathrm{H}^{p+q}\left(\mathcal{X}_{T}, \mathbb{C}\right)_{v}\left(\right.$ or $\left.F^{p} \mathrm{H}^{p+q+1}\left(\mathcal{Y}_{T}, \mathcal{X}_{T}, \mathbb{C}\right)\right)$ will be called algebraic if it lies in the image of $\overline{\operatorname{reg}}_{p, p-q}$.

0.3.2. Chow groups. In the case $p=q$ higher Chow groups are Chow groups and the regulator is the class map. I then write $\mathrm{CH}^{p}\left(\mathcal{X}_{T}\right)$ for $\mathrm{CH}^{p}\left(\mathcal{X}_{T}, 0\right), \mathrm{cl}_{p}$ for $\operatorname{reg}_{p, 0}$ and $\overline{\mathrm{cl}}_{p}$ for $\overline{\operatorname{reg}}_{p, 0}$

The following is a variation of my result in $\mathrm{O}$.

Theorem 2. Let $p, q, b$ and $c$ be positive integers such that $p+q=N, q \in\{[(N+$ $1) / 2], \ldots, N-1\}, b \geq 1$ and $c \leq b \frac{d^{N-q}}{(N-q) !}$. Assume $\phi(T) \subset \mathbb{P H}^{0}(Y, \mathcal{O}(d))$ lies in the locus parametrizing smooth hypersurfaces. Then then there is an integer $D$ depending only on $b, Y$ and $\mathcal{O}(1)$ such that for all $d \geq D$ the following holds.

If $N$ is even and $p=q=N / 2$ then the space $F^{p} \mathrm{H}^{p+q}\left(\mathcal{X}_{T}, \mathbb{C}\right)_{v}$ is spanned via $\overline{\mathrm{cl}}_{p}$ by classes of flat families of algebraic cycles in $\mathcal{X}_{T}$ of codimension $p$ and degree at most $b$.

If $q>N / 2$ then $F^{p} \mathrm{H}^{p+q}\left(\mathcal{X}_{T}, \mathbb{C}\right)_{v}=0$.

0.3.3. Higher Chow groups. I now fulfill the promise made in section 0.2 .2

Theorem 3. Let $p$ and $q$ be positive integers such that $p \geq q$ and $q \in\{[(N+1) / 2], \ldots, N-$ $1\}$. Assume that $Y \subset \mathbb{P H}^{0}(Y, \mathcal{O}(1))^{\vee}$ contains a codimension $q+1$ linear subvariety $V$ and let $T \subset \mathbb{P H}^{0}(Y, \mathcal{O}(d))$ be the subvariety parametrising smooth hypersurfaces containing $V$. Then for $d \gg 0$ the variety $T$ is of codimension $c \sim_{d \rightarrow \infty} \frac{d^{N-q}}{(N-q) !}$, and there is a higher cycle $Z \in \mathrm{CH}^{p}\left(\mathcal{X}_{T}, p-q\right)$ such that $\overline{\operatorname{reg}}_{p, p-q}(Z) \neq 0$.

Hence in this situation the bound on $c$ in Theorem 1 and its corollary are asymptotically optimal.

Note that the assumption on $Y$ holds for $Y=\mathbb{P}_{\mathbb{C}}^{N+1}$, which should be regarded as the main example.

0.3.4. Indecomposable Higher Chow groups. For all $i \in\{0, \ldots, p\}$ and $j \in\{0, \ldots, p-q\}$ there are natural product maps

$$
\psi_{i, j}: \mathrm{CH}^{p-i}\left(\mathcal{X}_{T}, p-q-j\right) \otimes \mathrm{CH}^{i}\left(\mathcal{X}_{T}, j\right) \rightarrow \mathrm{CH}^{p}\left(\mathcal{X}_{T}, p-q\right) .
$$


I say that a higher cycle in $\mathrm{CH}^{p}\left(\mathcal{X}_{T}, p-q\right)$ is decomposable if it belongs to the subgroup spanned by the images of the maps $\psi_{i, j}$ for $(i, j) \notin\{(0,0),(p, p-q)\}$, and that it is indecomposable otherwise.

The proof of Theorem 3 relies on an explicit construction of higher cycles in $\mathrm{CH}^{p}\left(\mathcal{X}_{T}, p-\right.$ q). More precisely, since $\mathrm{CH}^{1}\left(\mathcal{X}_{T}, 1\right) \simeq \mathrm{H}^{0}\left(\mathcal{X}_{T}, \mathcal{O}_{\mathcal{X}_{T}}^{*}\right) \simeq \mathrm{H}^{0}\left(T, \mathcal{O}_{T}^{*}\right)$, iterating the above map $p-q$ times I obtain the map

$$
\psi_{1,1}^{p-q}: \mathrm{CH}^{q}\left(\mathcal{X}_{T}\right) \otimes \mathrm{H}^{0}\left(T, \mathcal{O}_{T}^{*}\right)^{p-q} \rightarrow \mathrm{CH}^{p}\left(\mathcal{X}_{T}, p-q\right) .
$$

For $p>q$ the higher cycles in $\mathrm{CH}^{p}\left(\mathcal{X}_{T}, p-q\right)$ I construct are in the image of $\psi_{1,1}^{p-q}$. Since $\psi_{1,1}^{p-q}$ commutes to the restriction to a fiber, it follows that the restriction of these higher cycles to any fiber $X_{t}, t \in T$, is decomposable.

My result should be compared to Voisin's $\left[V 2\right.$, who studies the case $Y=\mathbb{P}_{\mathbb{C}}^{N+1}$, $p=N, q=N-1, d=2 N$ and $T \subset \mathbb{P H}^{0}(Y, \mathcal{O}(d))$ an open subset (this implies $c=$ $0)$. She constructs a family of higher cycles in $\mathrm{CH}^{N}\left(\mathcal{X}_{T}, 1\right)$ that limits the validity of Nori's connectivity theorem and whose restriction to a very general fiber $X_{t}$ for $t \in T$ is indecomposable. This suggests that for all $p, q$ such that $p+q \geq N$ and $p \geq q$ there might be higher cycles in $\mathrm{CH}^{p}\left(\mathcal{X}_{T}, p-q\right)$ for $c \sim_{d \rightarrow \infty} \frac{d^{N-q}}{(N-q) !}$ whose restriction to a very general fiber $X_{t}, t \in T$, is indecomposable. Supporting this intuition, I prove the following slightly weaker result for $Y=\mathbb{P}^{N+1}, N$ even, $q=N / 2$ and $p=q+1$.

Theorem 4. Assume $N$ even, $q=N / 2$ and that $Y \subset \mathbb{P H}^{0}(Y, \mathcal{O}(1))^{\vee}$ contains a codimension $q$ linear subvariety. Then for $d \gg 0$ there is a subvariety $U \subset \mathbb{P H}^{0}(Y, \mathcal{O}(d))$ of codimension $c \sim_{d \rightarrow \infty} 2 \frac{d^{N-q}}{(N-q) !}$, and a higher cycle $Z_{U} \in \mathrm{CH}^{q+1}\left(\mathcal{X}_{U}, 1\right)$ such that

$-\overline{\operatorname{reg}}_{q+1,1}\left(Z_{U}\right) \neq 0$, and

- the restriction of $Z_{U}$ to a very general fiber $X_{u}$ for $u \in U$ is indecomposable.

The cycle $Z_{U}$ is constructed explicitely and the proof of indecomposability relies on Theorem 2 .

I believe that Theorem 4 should generalize as follows.

Hope. Let $p$ and $q$ be positive integers such that $p>q$ and $q \in\{[(N+1) / 2], \ldots, N-$ $1\}$. Assume $Y=\mathbb{P}^{N+1}$. Then for $d \gg 0$ there is a subvariety $U \subset \mathbb{P H}^{0}(Y, \mathcal{O}(d))$ of codimension $c \sim_{d \rightarrow \infty}(p-q+1) \frac{d^{N-q}}{(N-q) !}$ and a higher cycle $Z_{U} \in \mathrm{CH}^{p}\left(\mathcal{X}_{U}, p-q\right)$ such that

$-\overline{\operatorname{reg}}_{p, p-q}\left(Z_{U}\right) \neq 0$, and

- the restriction of $Z_{U}$ to a very general fiber $X_{u}, u \in U$, is indecomposable.

Theorem 4 is the case $p=N / 2+1, q=N / 2$.

Note that $c \sim_{d \rightarrow \infty} 2 \frac{d^{N-q}}{(N-q) !}$ in Theorem四(and $c \sim_{d \rightarrow \infty}(p-q+1) \frac{d^{N-q}}{(N-q) !}$ in its conjectural generalisation) while Theorem 3 gives examples of decomposable cycles in $\mathrm{CH}^{p}\left(\mathcal{X}_{T}, p-q\right)$ with a non zero vanishing cohomology class for $c \sim_{d \rightarrow \infty} \frac{d^{N-q}}{(N-q) !}$. This suggests that the bound on $c$ in Theorem 4 may not be optimal.

Question. Is there an indecomposable higher cycle $Z_{U} \in \mathrm{CH}^{p}\left(\mathcal{X}_{U}, p-q\right)$ such that $\overline{\operatorname{reg}}_{p, p-q}\left(Z_{U}\right) \neq 0$ for $c \sim_{d \rightarrow \infty} \frac{d^{N-q}}{(N-q) !}$ ? 
0.4. Algebraicity conjecture. The results of Section 0.3 suggest that for $c$ slightly bigger than the bound $\frac{d^{N-q}}{(N-q) !}$ given by Theorem 1 the space $F^{p} \mathrm{H}^{p+q}\left(\mathcal{X}_{T}, \mathbb{Q}\right)_{v}$ should be algebraic.

More precisely, I believe the following should hold

Conjecture 1. For all integers $b \in \mathbb{N}^{*}$ and $p \in \mathbb{N}$ there is an integer $D$ depending only on $b, p, Y$ and on $\mathcal{O}(1)$, such that for all $d \geq D$ and $0 \leq q \leq N-1$, if

$$
c \leq b \frac{d^{N-q}}{(N-q) !}
$$

then the map $\overline{\operatorname{reg}}_{p, p-q}: \mathrm{CH}^{p}\left(\mathcal{X}_{T}, p-q\right) \otimes \mathbb{C} \rightarrow F^{p} \mathrm{H}^{p+q}\left(\mathcal{X}_{T}, \mathbb{C}\right)_{v}$ is surjective.

By convention, $\mathrm{CH}^{p}\left(\mathcal{X}_{T}, p-q\right)=0$ for $p<q$.

By Theorem 2, Conjecture 1 holds for $p+q=N, q \geq N / 2$ provided that $\phi(T) \subset$ $\mathbb{P H}^{0}(Y, \mathcal{O}(d))$ lies in the locus parametrizing smooth hypersurfaces.

Mimicking Nori's argument, it is easy to see that Conjecture 1 implies the following purely topological statement.

Conjecture 2. For every positive integer $b$ there is an integer $D$ depending only on $b$, $Y$ and $\mathcal{O}(1)$ such that for all $d \geq D$ and $k \in\{N, \ldots, 2 N-1\}$ and for

$$
c \leq b \frac{d^{N-[k / 2]}}{(N-[k / 2]) !}
$$

- if $k$ is even then the map $\overline{\mathrm{cl}}_{k / 2}: \mathrm{CH}^{k / 2}\left(\mathcal{X}_{T}\right) \otimes \mathbb{C} \rightarrow F^{k / 2} \mathrm{H}^{k}\left(\mathcal{X}_{T}, \mathbb{C}\right)_{v}$ is surjective;

- if $k$ is odd then the maps

$$
\begin{aligned}
& \overline{\operatorname{reg}}_{(k+1) / 2,1}: \mathrm{CH}^{(k+1) / 2}\left(\mathcal{X}_{T}, 1\right) \otimes \mathbb{C} \rightarrow \mathrm{H}^{k}\left(\mathcal{X}_{T}, \mathbb{C}\right)_{v}, \quad \text { and } \\
& \psi \circ \overline{\operatorname{reg}}_{(k+1) / 2,1}: \mathrm{CH}^{(k+1) / 2}\left(\overline{\mathcal{X}_{T}}, 1\right) \otimes \mathbb{C} \rightarrow \mathrm{H}^{k}\left(\overline{\mathcal{X}_{T}}, \mathbb{C}\right)_{v} \rightarrow \mathrm{H}^{k}\left(\mathcal{X}_{T}, \mathbb{C}\right)_{v} \\
& \text { generate } \mathrm{H}^{k+1}\left(\mathcal{Y}_{T}, \mathcal{X}_{T}, \mathbb{C}\right)_{v} .
\end{aligned}
$$

Here $\overline{\mathcal{X}_{T}}$ is the variety isomorphic to $\mathcal{X}_{T}$ as a real manifold and endowed with the opposite complex structure and $\psi$ is the geometric frobenus.

Note that for $p+q<N$ Conjecture 1 and for $k<N$ Conjecture 2 are trivially true since the target cohomology spaces vanish ( $c f$. section 0.2.1).

For $k$ even Theorem 1 implies that in the numerical situation of Conjecture 2 , one has $F^{k / 2+1} \mathrm{H}^{k}\left(\mathcal{Y}_{T}, \mathcal{X}_{T}, \mathbb{C}\right)=0$, hence by a mixed Hodge structures argument the space $\mathrm{H}^{k}\left(\mathcal{Y}_{T}, \mathcal{X}_{T}, \mathbb{C}\right)$ is of pure Hodge type $(k / 2, k / 2)$ (like in $\mathbb{N o}$, section 0.2$)$, hence the subspace $\mathrm{H}^{k}\left(\mathcal{X}_{T}, \mathbb{C}\right)_{v}$ is a sub-Hodge structure of pure Hodge type $(k / 2, k / 2)$. Hence for $k$ even Conjecture 2 is implied by Hodge Conjecture.

0.5. Summary. The figures summarise the results. Namely, for $c \leq b \frac{d^{N-q}}{(N-q) !}$

a : $F^{p} \mathrm{H}^{p+q+1}\left(\mathcal{Y}_{T}, \mathcal{X}_{T}, \mathbb{C}\right)=0$ for any $c$ by section 0.2 .1

b : $F^{p} \mathrm{H}^{p+q+1}\left(\mathcal{Y}_{T}, \mathcal{X}_{T}, \mathbb{C}\right)=0$ for $c \leq(1-\varepsilon) \frac{d^{N-q}}{(N-q) !}$ by Theorem 1 and $F^{p} \mathrm{H}^{p+q}\left(\mathcal{X}_{T}, \mathbb{C}\right)_{v}$ is spanned by classes of flat families of algebraic cycles of codimension $N / 2$ and degree at most $b$ for $c \leq b \frac{d^{N-q}}{(N-q) !}$ by Theorem 2] as predicted by Conjecture 1, 


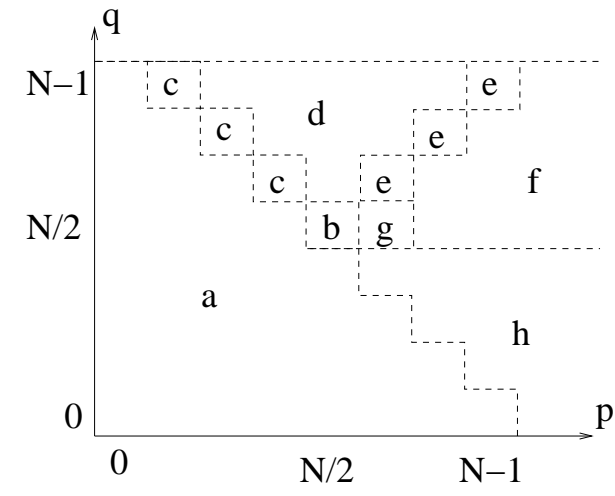

$\mathrm{N}$ even

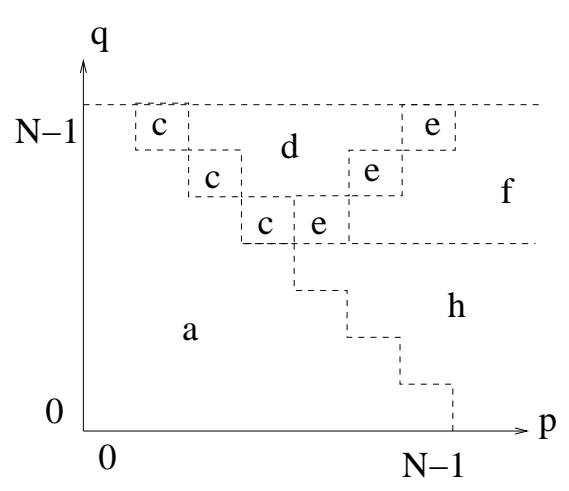

$\mathrm{N}$ odd

c : $F^{p} \mathrm{H}^{p+q+1}\left(\mathcal{Y}_{T}, \mathcal{X}_{T}, \mathbb{C}\right)=0$ for $c \leq b \frac{d^{N-q}}{(N-q) !}$ by Theorem 2 .

$\mathrm{d}: F^{p} \mathrm{H}^{p+q+1}\left(\mathcal{Y}_{T}, \mathcal{X}_{T}, \mathbb{C}\right)=0$ for $c \leq(1-\varepsilon) \frac{d^{N-q}}{(N-q) !}$ by Theorem $\square$ and one should still have $F^{p} \mathrm{H}^{p+q}\left(\mathcal{X}_{T}, \mathbb{C}\right)_{v}=0$ for $c \leq b \frac{d^{N-q}}{(N-q) !}$ by Conjecture 1

e : $F^{p} \mathrm{H}^{p+q+1}\left(\mathcal{Y}_{T}, \mathcal{X}_{T}, \mathbb{C}\right)=0$ for $c \leq(1-\varepsilon) \frac{d^{N-q}}{(N-q) !}$ by Theorem 1 and $F^{p} \mathrm{H}^{p+q}\left(\mathcal{X}_{T}, \mathbb{C}\right)_{v}$ should be spanned by $\overline{\mathrm{cl}}_{p}\left(\mathrm{CH}^{p}\left(\mathcal{X}_{T}\right)\right)$ for $c \leq b \frac{d^{N-q}}{(N-q) !}$ by Conjecture 1] by Theorem 3 there is a variety $Y$ such that $\overline{\mathrm{cl}}_{p}\left(\mathrm{CH}^{p}\left(\mathcal{X}_{T}\right)\right) \neq 0$ for $c \sim \frac{d^{N-q}}{(N-q) !}$.

f : $F^{p} \mathrm{H}^{p+q+1}\left(\mathcal{Y}_{T}, \mathcal{X}_{T}, \mathbb{C}\right)=0$ for $c \leq(1-\varepsilon) \frac{d^{N-q}}{(N-q) !}$ by Theorem 1 and $F^{p} \mathrm{H}^{p+q}\left(\mathcal{X}_{T}, \mathbb{C}\right)_{v}$ should be spanned by $\overline{\operatorname{reg}}_{p, p-q} \mathrm{CH}^{p}\left(\mathcal{X}_{T}, p-q\right)$ for $c \leq b \frac{d^{N-q}}{(N-q) !}$ by Conjecture 1 by Theorem 3 there is a variety $Y$ such that $\overline{\operatorname{reg}}_{p, p-q} \mathrm{CH}^{p}\left(\mathcal{X}_{T}, p-q\right) \neq 0$ for $c \sim \frac{d^{N-q}}{(N-q) !}$ and by Conjecture,there should be an indecomposable higher cycle in $\mathrm{CH}^{p}\left(\mathcal{X}_{T}, p-q\right)$ with a non zero image under $\overline{\operatorname{reg}}_{p, p-q}$ for $c \sim(p-q+1) \frac{d^{N-q}}{(N-q) !}$.

$\mathrm{g}$ : as in $\mathrm{f}$; by Theorem 4 there is a variety $Y$ and an indecomposable higher cycle in $\mathrm{CH}^{p}\left(\mathcal{X}_{T}, p-q\right)$ with a non zero image under $\overline{\operatorname{reg}}_{p, p-q}$ for $c \sim 2 \frac{d^{N-q}}{(N-q) !}$.

$\mathrm{h}: F^{p} \mathrm{H}^{p+q+1}\left(\mathcal{Y}_{T}, \mathcal{X}_{T}, \mathbb{C}\right)=0$ for $c \leq(1-\varepsilon) \frac{d^{N-q}}{(N-q) !}$ by Theorem 1 and $F^{p} \mathrm{H}^{p+q}\left(\mathcal{X}_{T}, \mathbb{C}\right)_{v}$ should be spanned by $\overline{\operatorname{reg}}_{p, p-q}\left(\mathrm{CH}^{p}\left(\mathcal{X}_{T}, p-q\right)\right)$ for $c \leq b \frac{d^{N-q}}{(N-q) !}$ by Conjecture 1 Here the situation is somewhat mysterious since I do not have any example of a non-zero element in $\mathrm{CH}^{p}\left(\mathcal{X}_{T}, p-q\right)$.

\section{Proof of Theorem 1 and of its Corollary}

1.1. Reduction to the local case. A straightforward adaptation of Nori's argument shows that if $\mathrm{H}^{q+1}\left(Y_{t}, \Omega_{\mathcal{Y}_{T}, \mathcal{X}_{T} \mid Y_{t}}^{p}\right)=0$ for all $t \in T$ then $\mathrm{H}^{q+1}\left(\mathcal{Y}_{T}, \Omega_{\mathcal{Y}_{T}, \mathcal{X}_{T}}^{p}\right)=0$ ([No], Lemma 2.1). This shows that Theorem 1 is implied by the following purely local-analytic statement.

Proposition 1.2. For all $\varepsilon \in \mathbb{R}_{+}^{*}$ there is an integer $D$ depending only on $p, \varepsilon, Y$ and on $\mathcal{O}(1)$ such that

$$
\mathrm{H}^{q+1}\left(Y_{t}, \Omega_{\mathcal{Y}_{T}, \mathcal{X}_{T} \mid Y_{t}}^{p}\right)=0
$$


for all integers $d \geq D, q \in\{-1, \ldots, N-1\}$, for all smooth analytic varieties $T$ endowed with a morphism $\phi: T \rightarrow \mathrm{H}^{0}(Y, \mathcal{O}(d))$ such that

- for all $t \in T$ the cokernel of $d \phi(t)$ is of dimension at most $(1-\varepsilon) \frac{d^{N-q}}{(N-q) !}$ and

$-\mathcal{X}_{T}$ is smooth.

1.3. Proof of proposition 1.2. Since the proof of proposition 1.2 proceeds by induction on several parameters, it is convenient to introduce the following notation. For a fixed positive integer $d$ and a projective space $\mathbb{P}_{\mathbb{C}}^{A}$ I say that assertion $\mathcal{H}(Y, p, q, c, a)$ holds for the smooth projective variety $Y \subset \mathbb{P}_{\mathbb{C}}^{A}$ of dimension $N+1>0$ and for the integers $(p, q, c, a) \in \mathbb{N} \times\{-1, \ldots, N-1\} \times \mathbb{N} \times \mathbb{Z}$ if

$$
\mathrm{H}^{q+1}\left(Y_{t}, \Omega_{\mathcal{Y}_{T}, \mathcal{X}_{T} \mid Y_{t}}^{p} \otimes \mathcal{O}\left(a^{\prime}\right)\right)=0
$$

for all $a^{\prime} \leq a$, for all smooth analytic varieties $T$ endowed with a morphism $\phi: T \rightarrow$ $\mathrm{H}^{0}(Y, \mathcal{O}(d))$ such that $\mathcal{X}_{T}$ is smooth, and for all $t \in T$ such that the cokernel of $d \phi(t)$ is of dimension at most $c$.

The proof relies on the following four lemmas.

Recall ( $c f$. [G1]) that for any positive integer $c$, a $d$-decomposition of $c$ is the unique series $\left(c_{d}, \ldots, c_{r}\right)$ such that $r \in\{1, \ldots, d\}, c_{d}>\cdots>c_{r} \geq r$ and

$$
c=\left(\begin{array}{c}
c_{d} \\
d
\end{array}\right)+\cdots+\left(\begin{array}{c}
c_{r} \\
r
\end{array}\right) \text {. }
$$

Let

$$
c_{<d>}=\left(\begin{array}{c}
c_{d}-1 \\
d
\end{array}\right)+\cdots+\left(\begin{array}{c}
c_{r}-1 \\
r
\end{array}\right),
$$

with the convention $\left(\begin{array}{l}\alpha \\ \beta\end{array}\right)=0$ for $\alpha<\beta$.

The asympotic behaviour of the function $c \mapsto c_{<d>}$ is described by the following lemma.

Lemma 1.3.1. For all integers $M \geq 2$ there exists a function $\left.f_{M}:\right] 0,1[\times \mathbb{N} \rightarrow] 0,1[$, $(\varepsilon, d) \mapsto \varepsilon^{\prime}$ such that for all $\left.\varepsilon \in\right] 0,1\left[\varepsilon^{\prime} \rightarrow \varepsilon^{\frac{M-1}{M}}\right.$ for $d \rightarrow \infty$, and such that for all nonnegative integers

one has

$$
c \leq(1-\varepsilon) \frac{d^{M}}{M !}
$$

$$
c_{<d>} \leq\left(1-\varepsilon^{\prime}\right) \frac{d^{M-1}}{(M-1) !} .
$$

The following lemma is the key ingedient of my proof. It relies on Green's hyperplane section theorem G1].

Lemma 1.3.2. Let $Y^{\prime}$ be a generic hyperplane section of $Y$. Then for $q \leq N-2$

$$
\left.\begin{array}{r}
\mathcal{H}\left(Y^{\prime}, p, q, c_{<d>}, a\right) \\
\mathcal{H}\left(Y^{\prime}, p-1, q, c_{<d>}, a-1\right)
\end{array}\right\} \Rightarrow \mathcal{H}(Y, p, q, c, a) .
$$

By convention, assumption $\mathcal{H}\left(Y^{\prime},-1, q, c_{<d>}, a-1\right)$ holds.

The last two lemmas will only be used for $q=N-1$. Both rely on an idea of Green (G2, p. 193-194). 
Lemma 1.3.3. Assume $c>0$. Then

$$
\left.\begin{array}{l}
\mathcal{H}(Y, p+1, q, c-1, a) \\
\mathcal{H}(Y, p+1, q-1, c, a)
\end{array}\right\} \Rightarrow \mathcal{H}(Y, p, q, c, a)
$$

By convention, assumption $\mathcal{H}(Y, p+1,-2, c, a)$ holds.

The last lemma has been proven by Nori for $a=0$ ( $[\mathbf{N o}$, remark 3.10); the general case involves no new ideas. See also $[\mathbb{N}]$ for computations of an explicit bound for $s$.

Lemma 1.3.4. There is an integer $s$ depending only on $Y$ and $\mathcal{O}(1)$ such that for all $d \geq p+a+s$ the assertion $\mathcal{H}(Y, p, q, 0, a)$ holds for all $q \in\{-1, \ldots, N-1\}$.

Lemma 1.3.4 will only be used for $q=N-1$.

1.3.5. Proof of proposition 1.2. Postponing the proofs of the above Lemmas, I now show how they imply proposition 1.2

I show more generally that for all $\varepsilon \in] 0,1[$ there is an integer $D$ depending only on $\varepsilon$, $p, a$ and $Y$ such that assertion $\mathcal{H}(Y, p, q, c, a)$ holds for all $d \geq D, q \in\{-1, \ldots, N-1\}$ and $c \leq(1-\varepsilon) \frac{d^{N-q}}{(N-q) !}$. The case $a=0$ is the proposition 1.2

I proceed by induction on $N-q, c$ and $N$.

Assume $N-q=1$. The condition on $c$ simplifies to $c \leq(1-\varepsilon) d$. Choose $D$ such that $\varepsilon D \geq p+a+s$. Thus for $d \geq D$ one has $d \geq(1-\varepsilon) d+\varepsilon D \geq c+p+a+s$.

The case $c=0, N-q=1$ follows from Lemma 1.3.4

The case $c>0, N=0$ and $q=-1$ follows from Lemma 1.3.3 by increasing induction on $c$. For the case $c>0, N-q=1$ let $Y^{\prime}$ be a generic hyperplane section of $Y$. Lemmas 1.3 .2 and 1.3 .3 provide successive implications

$$
\left.\left.\begin{array}{l}
\mathcal{H}\left(Y^{\prime}, p+1, q-1, c_{<d>}, a\right) \\
\mathcal{H}\left(Y^{\prime}, p, q-1, c_{<d>}, a-1\right)
\end{array}\right\} \Rightarrow \begin{array}{r}
\mathcal{H}(Y, p+1, q, c-1, a) \\
\mathcal{H}(Y, p+1, q-1, c, a)
\end{array}\right\} \Rightarrow \mathcal{H}(Y, p, q, c, a) .
$$

Assertion $\mathcal{H}(Y, p, q, c, a)$ follows by increasing induction on both $c$ and $N$.

Assume $N-q>1$ and let $Y^{\prime}$ be a generic hyperplane section of $Y$. By induction on $N$ and by Lemma 1.3.1 applied to $M=N-q$, assertions $\mathcal{H}\left(Y^{\prime}, p, q, c_{<d>}, a\right)$ and $\mathcal{H}\left(Y^{\prime}, p-1, q, c_{<d>}, a-1\right)$ hold. Hence by Lemma 1.3.2 assertion $\mathcal{H}(Y, p, q, c, a)$ holds.

\subsection{Proof of the Lemmas.}

1.4.1. Proof of Lemma 1.3.1. Let $e$ be the smallest positive integer such that

$$
(1-\varepsilon) \frac{d^{M}}{M !} \leq\left(\begin{array}{c}
M+d \\
d
\end{array}\right)-\left(\begin{array}{c}
M+e \\
e
\end{array}\right)
$$

for all $q \in\{0, \ldots, N-1\}$. One has $e \sim \varepsilon^{1 / M} d$ for $d \rightarrow \infty$.

The map $c \mapsto c_{<d>}$ is increasing. Thanks to the identities

$$
\begin{aligned}
\left(\begin{array}{c}
M+d \\
d
\end{array}\right)-\left(\begin{array}{c}
M+e \\
e
\end{array}\right) & =\sum_{i=1}^{d-e}\left(\begin{array}{c}
M+d-i \\
d-i+1
\end{array}\right) \\
\left(\begin{array}{c}
M-1+d \\
d
\end{array}\right)-\left(\begin{array}{c}
M-1+e \\
d-e
\end{array}\right) & =\sum_{i=1}^{d-e}\left(\begin{array}{c}
M-1+d-i \\
d-i+1
\end{array}\right)
\end{aligned}
$$


One gets

$$
c_{<d>} \leq\left(\begin{array}{c}
M-1+d \\
d
\end{array}\right)-\left(\begin{array}{c}
M-1+e \\
e
\end{array}\right)
$$

The term on the right is asymptotically equivalent to $\varepsilon^{\frac{M-1}{M}} \frac{d^{M-1}}{(M-1) !}$ for $d \rightarrow \infty$. Define $\left.\varepsilon^{\prime} \in\right] 0,1\left[\right.$ by $\varepsilon^{\prime}=\varepsilon^{\frac{M-1}{M}}$ if $\frac{d^{M-1}}{(M-1) !} \geq\left(\begin{array}{c}M-1+d \\ d\end{array}\right)-\left(\begin{array}{c}M-1+e \\ e\end{array}\right)$ and by the formula

$$
\left(1-\varepsilon^{\prime}\right) \frac{d^{M-1}}{(M-1) !}=\left(\begin{array}{c}
M-1+d \\
d
\end{array}\right)-\left(\begin{array}{c}
M-1+e \\
e
\end{array}\right)
$$

otherwise. Thus $\left.\varepsilon^{\prime} \in\right] 0,1\left[\right.$ and $\varepsilon^{\prime} \sim \varepsilon^{\frac{M-1}{M}}$ for $d \rightarrow \infty$.

1.4.2. Proof of Lemma 1.3.2. Let $i: Y^{\prime} \rightarrow Y$ be the projective embedding, let $\mathcal{Y}_{T}^{\prime}=Y^{\prime} \times T$ and let $\mathcal{X}_{T}^{\prime}=\mathcal{X}_{T} \times \mathcal{Y}_{T} \mathcal{Y}_{T}^{\prime}$. Fix $t \in T$.

Since $Y^{\prime}$ is generic, one may assume that $\mathcal{X}_{T}^{\prime}$ is smooth and that $X_{t}$ meets $Y_{t}^{\prime}$ transversally in $Y_{t}$. Restricting $T$ if necessary, one may further assume that for all $u \in T, X_{u}$ meets $Y_{u}^{\prime}$ transversally in $Y_{u}$. Hence there is a well-defined map $\phi^{\prime}: T \rightarrow \mathbb{P H}^{0}\left(Y^{\prime}, \mathcal{O}(d)\right)$, constructed as follows.

Let $p_{Y}: \mathrm{H}^{0}(Y, \mathcal{O}(d)) \backslash\{0\} \rightarrow \mathbb{P H}^{0}(Y, \mathcal{O}(d))$ be the natural projection and let

$$
p_{Y}^{*} \phi: T \times_{\mathbb{P} \mathrm{H}^{0}(Y, \mathcal{O}(d))} \mathrm{H}^{0}(Y, \mathcal{O}(d)) \rightarrow \mathrm{H}^{0}(Y, \mathcal{O}(d))
$$

be the map deduced from $\phi$ by base change. Then $\phi^{\prime}$ is defined by the map

$$
p_{Y^{\prime}}^{*} \phi^{\prime}: T \times_{\mathbb{P H} H^{0}(Y, \mathcal{O}(d))} \mathrm{H}^{0}(Y, \mathcal{O}(d)) \rightarrow \mathrm{H}^{0}\left(Y^{\prime}, \mathcal{O}(d)\right)
$$

obtained by composing $p_{Y}^{*} \phi$ with the linear projection $\mathrm{H}^{0}(Y, \mathcal{O}(d)) \rightarrow \mathrm{H}^{0}\left(Y^{\prime}, \mathcal{O}(d)\right)$ which maps to 0 the polynomials vanishing on $Y^{\prime}$.

Let $E=p_{Y}^{*} \operatorname{Im}(d \phi(t))$, resp. $E^{\prime}=p_{Y^{\prime}}^{*} \operatorname{Im}\left(d \phi^{\prime}(t)\right)$. Let

$$
\begin{aligned}
c=\operatorname{dim} \operatorname{coker}(d \phi(t)) & =\operatorname{codim}\left(E, \mathrm{H}^{0}(Y, \mathcal{O}(d))\right), \quad \text { resp. } \\
c^{\prime}=\operatorname{dim} \text { coker }\left(d \phi^{\prime}(t)\right) & =\operatorname{codim}\left(E^{\prime}, \mathrm{H}^{0}\left(Y^{\prime}, \mathcal{O}(d)\right)\right) .
\end{aligned}
$$

Let $S=\bigoplus_{\delta \in \mathbb{N}} S_{\delta}$, resp. $\quad S^{\prime}=\bigoplus_{\delta \in \mathbb{N}} S_{\delta}^{\prime}$ denote the homogenous polynomial ring of $\mathrm{H}^{0}(Y, \mathcal{O}(1))$, resp. $\mathrm{H}^{0}\left(Y^{\prime}, \mathcal{O}(1)\right)$. Let $I \subset S$, resp. $I^{\prime} \subset S^{\prime}$ denote the homogenous ideal of $Y$, resp. $Y^{\prime}$. One has $c=\operatorname{codim}\left(E+I_{d}, S_{d}\right)$, resp. $c^{\prime}=\operatorname{codim}\left(E^{\prime}+I_{d}^{\prime}, S_{d}^{\prime}\right)$. Since $Y^{\prime} \subset Y$ is generic, Green's hyperplane theorem [G1] asserts that

$$
c^{\prime} \leq c_{<d>}
$$

Hence the cokernel of $d \phi^{\prime}(t)$ is of dimension at most $c_{<d>}$.

The sheaf $\Omega_{\mathcal{Y}_{T}, \mathcal{X}_{T} \mid \mathcal{Y}_{T}^{\prime}}^{p}$ fits into the short exact sequence

$$
0 \longrightarrow \Omega_{\mathcal{Y}_{T}^{\prime}, \mathcal{X}_{T}^{\prime}}^{p-1} \otimes \mathcal{O}_{Y}^{\prime}(-1) \stackrel{d H \wedge}{\longrightarrow} \Omega_{\mathcal{Y}_{T}, \mathcal{X}_{T} \mid \mathcal{Y}_{T}^{\prime}}^{p} \longrightarrow \Omega_{\mathcal{Y}_{T}^{\prime}, \mathcal{X}_{T}^{\prime}}^{p} \longrightarrow 0
$$

where $H \in \mathrm{H}^{0}(Y, \mathcal{O}(1))$ is an equation of $Y^{\prime}$. Restricting this sequence to $Y_{t}^{\prime}$ and tensorising it by $\mathcal{O}_{Y}^{\prime}(a)$ one gets the associated long exact sequence

$$
\begin{aligned}
\mathrm{H}^{q+1}\left(Y_{t}^{\prime}, \Omega_{\mathcal{Y}_{T}^{\prime}, \mathcal{X}_{T}^{\prime} \mid Y_{t}^{\prime}}^{p-1} \otimes \mathcal{O}_{Y}^{\prime}(a-1)\right) \stackrel{d H \wedge}{\longrightarrow} & \mathrm{H}^{q+1}\left(Y_{t}^{\prime}, \Omega_{\mathcal{Y}_{T}, \mathcal{X}_{T} \mid Y_{t}^{\prime}}^{p} \otimes \mathcal{O}_{Y}^{\prime}(a)\right) \longrightarrow \\
& \longrightarrow \mathrm{H}^{q+1}\left(Y_{t}^{\prime}, \Omega_{\mathcal{Y}_{T}^{\prime}, \mathcal{X}_{T}^{\prime} \mid Y_{t}^{\prime}}^{\longrightarrow} \mathcal{O}_{Y}^{\prime}(a)\right) .
\end{aligned}
$$


The term on the left vanishes by assumption $\mathcal{H}\left(Y^{\prime}, p-1, q, c_{<d>}, a-1\right)$ and the term on the right vanishes by assumption $\mathcal{H}\left(Y^{\prime}, p, q, c_{<d>}, a\right)$. Hence the middle term vanishes as well: one has

$$
\mathrm{H}^{q+1}\left(Y_{t}^{\prime}, \Omega_{\mathcal{Y}_{T}, \mathcal{X}_{T} \mid Y_{t}^{\prime}}^{p} \otimes \mathcal{O}_{Y}^{\prime}(a)\right)=0 .
$$

For any coherent sheaf $\mathcal{F}$ on $Y_{t}$ there is a short exact sequence

$$
0 \longrightarrow \mathcal{F} \otimes \mathcal{O}(-1) \stackrel{H}{\longrightarrow} \mathcal{F} \longrightarrow i_{*} i^{*} \mathcal{F} \longrightarrow 0
$$

Take $\mathcal{F}=\Omega_{\mathcal{Y}_{T}, \mathcal{X}_{T} \mid Y_{t}}^{p} \otimes \mathcal{O}_{Y}(a)$ and consider the associated long exact sequence

$$
\begin{aligned}
\mathrm{H}^{q+1}\left(Y_{t}, \Omega_{\mathcal{Y}_{T}, \mathcal{X}_{T} \mid Y_{t}}^{p} \otimes \mathcal{O}_{Y}(a-1)\right) \stackrel{H}{\longrightarrow} \mathrm{H}^{q+1}\left(Y_{t}, \Omega_{\mathcal{Y}_{T}, \mathcal{X}_{T} \mid Y_{t}}^{p} \otimes \mathcal{O}_{Y}(a)\right) \\
\longrightarrow \mathrm{H}^{q+1}\left(Y_{t}^{\prime}, \Omega_{\mathcal{Y}_{T}, \mathcal{X}_{T} \mid Y_{t}^{\prime}}^{p} \otimes \mathcal{O}_{Y}^{\prime}(a)\right) .
\end{aligned}
$$

The term on the right vanishes by (11). Since $q \in\{0, \ldots, N-1\}$, by increasing induction on $a$ the term on the right vanishes as well, the case $a \ll 0$ following from Serre's vanishing theorem. Hence the term in the middle vanishes.

1.4.3. Proof of Lemma 1.3.3. Since the assertion depends only on the tangent space of $T$ at $t$, one may replace $T$ by a small open neighbourhood of $t$ in the tangent space of $T$ at $t$; hence one may assume $T=B \times S$, where $B \subset \mathbb{P H}^{0}(Y, \mathcal{O}(d))$ is an open neighbourghood of $\phi(t)$ of codimension at most $c$ and $S$ is an open ball.

Let $B^{\prime} \subset \mathbb{P H}^{0}(Y, \mathcal{O}(d))$ be a smooth analytic variety containing $B$ as a codimension 1 subvariety, and let $T^{\prime}=B^{\prime} \times S$. Then $\mathcal{Y}_{T} \subset \mathcal{Y}_{T^{\prime}}$ is a smooth codimension 1 subvariety and one has the following short exact sequence of sheaves on $\mathcal{Y}_{T}$ :

$$
0 \longrightarrow \Omega_{\mathcal{Y}_{T}, \mathcal{X}_{T}}^{p} \longrightarrow \Omega_{\mathcal{Y}_{T^{\prime}}, \mathcal{X}_{T^{\prime}} \mid \mathcal{Y}_{T}}^{p+1} \longrightarrow \Omega_{\mathcal{Y}_{T}, \mathcal{X}_{T}}^{p+1} \longrightarrow 0 .
$$

Restrict this sequence to $Y_{t}$, tensorise it with $\mathcal{O}(a)$ and consider the associated long exact sequence

$\mathrm{H}^{q}\left(Y_{t}, \Omega_{\mathcal{Y}_{T}, \mathcal{X}_{T} \mid Y_{t}}^{p+1} \otimes \mathcal{O}(a)\right) \longrightarrow \mathrm{H}^{q+1}\left(Y_{t}, \Omega_{\mathcal{Y}_{T}, \mathcal{X}_{T} \mid Y_{t}}^{p} \otimes \mathcal{O}(a)\right) \longrightarrow \mathrm{H}^{q+1}\left(Y_{t}, \Omega_{\mathcal{Y}_{T^{\prime}}, \mathcal{X}_{T^{\prime}} \mid Y_{t}}^{p+1} \otimes \mathcal{O}(a)\right)$

The terms on the right and on the left vanish by assumption, hence the term in the middle vanishes as well.

\section{Proof of Theorem 2}

Consider the exact sequence

$$
R^{k} \pi_{*} \mathbb{C} \rightarrow R^{k} \pi_{*}\left(j_{T *} \mathbb{C}\right) \rightarrow R^{k} \pi_{*}\left(\mathbb{C} \rightarrow j_{T *} \mathbb{C}\right) \rightarrow R^{k+1} \pi_{*} \mathbb{C} \rightarrow R^{k+1} \pi_{*}\left(j_{T *} \mathbb{C}\right)
$$

and let $\mathcal{H}_{\mathcal{X}_{T}, v}^{k}=\operatorname{coker}\left(R^{k} \pi_{*} \mathbb{C} \rightarrow R^{k} \pi_{*}\left(j_{T *} \mathbb{C}\right)\right)$ denote the locally trivial subsheaf of $R^{k} \pi_{Y *}\left(\mathbb{C} \rightarrow j_{T *} \mathbb{C}\right)$ whose fiber at any point $t \in T$ is canonically isomorphic to $\mathrm{H}^{k}\left(X_{t}, \mathbb{C}\right)_{v}$. Let $F^{p} \mathrm{H}^{0}\left(T, \mathcal{H}_{\mathcal{X}_{T}, v}^{k}\right)$ denote the space of sections of $\mathcal{H}_{\mathcal{X}_{T}, v}^{k}$ whose fiber at any point $t \in T$ belongs to $F^{p} \mathrm{H}^{k}\left(X_{t}, \mathbb{C}\right)_{v}$.

In [O] I have shown 
Theorem $(\underline{\mathrm{O}})$. For all integers $b \in \mathbb{N}^{*}$ there is an integer $D$ depending only on $b, Y$ and on $\mathcal{O}(1)$, such that for all $d \geq D$, for all $q \in\{[(N+1) / 2], \ldots, N-1\}$, for all

$$
c \leq b \frac{d^{N-q}}{(N-q) !}
$$

for all codimension c algebraic varieties $T \subset \mathbb{P H}^{0}(Y, \mathcal{O}(d))$ lying in the locus parametrizing smooth hypersurfaces and for all $t \in T$, the following holds.

If $N$ is even and $q=N / 2$ then the space $F^{N-q} \mathrm{H}^{0}\left(T, \mathcal{H}_{\mathcal{X}_{T}, v}^{N}\right)$ is spanned by families of algebraic cycles of codimension $q$ and degree at most $b$.

$$
\text { If } q>N / 2 \text { then } F^{N-q} \mathrm{H}^{0}\left(T, \mathcal{H}_{\mathcal{X}_{T}, v}^{N}\right)=0 \text {. }
$$

The proof of $[\mathrm{O}$ gives in fact a stronger result, namely one can replace the subspace $T \subset$ $\mathbb{P H}^{0}(Y, \mathcal{O}(d))$ by any algebraic variety $T$ endowed with a morphism $\phi: T \rightarrow \mathbb{P H}^{0}(Y, \mathcal{O}(d))$, such that

- $\phi(T)$ lies in the locus parametrizing smooth hypersurfaces and

- for all $t \in T$ the cokernel of the differential $d \phi(t): T_{T, t} \rightarrow T_{\mathbb{P H}^{0}(Y, \mathcal{O}(d)), \phi(t)}$ is of dimension at most $c$;

This stronger version of the theorem of [O] is equivalent to Theorem 2, Indeed, one only needs to show

$$
F^{N-q} \mathrm{H}^{N}\left(\mathcal{X}_{T}, \mathbb{C}\right)_{v} \simeq F^{N-q} \mathrm{H}^{0}\left(T, \mathcal{H}_{\mathcal{X}_{T}, v}^{N}\right) .
$$

But the Lefschetz hyperplane Theorem and the Leray spectral sequence give a canonical isomorphism

$$
\mathrm{H}^{N}\left(\mathcal{X}_{T}, \mathbb{C}\right)_{v} \simeq \mathrm{H}^{0}\left(T, \mathcal{H}_{\mathcal{X}_{T}, v}^{N}\right)
$$

Since for any $t \in T$, the restriction map $\mathrm{H}^{N}\left(\mathcal{X}_{T}, \mathbb{C}\right)_{v} \rightarrow \mathrm{H}^{N}\left(\mathcal{X}_{t}, \mathbb{C}\right)_{v}$ is a homomorphism of Hodge structures, hence is strict, the result follows.

\section{Proof of Theorem 3}

Since $q \geq N / 2$, the generic hypersurface of degree $d$ containing $V$ is smooth; let $T \subset$ $\mathbb{P H}^{0}(Y, \mathcal{O}(d))$ be a smooth open affine subvariety of the space of all smooth hypersurfaces containing $V$. One has $c \sim_{d \rightarrow \infty} \frac{d^{N-q}}{(N-q) !}$.

For all $i \in\{1, \ldots, p-q\}$ choose hyperplane sections $H_{i} \subset T$ in general position and and let $f_{i} \in \mathrm{H}^{0}\left(T, \mathcal{O}_{T}\right)$ be the equations of $H_{i}$; Let $P_{i}=\bigcap_{j>i} H_{j}, Q_{i}=\bigcup_{j<i} H_{j}$ and $T_{i}=P_{i} \backslash\left(P_{i} \cap Q_{i}\right)$. For $j \leq i$ the functions $f_{j}$ do not vanish on $T_{i}$, hence belong to $\mathrm{H}^{0}\left(T_{i}, \mathcal{O}_{T_{i}}^{*}\right)$ and there are well-defined higher cycles in $\mathrm{CH}^{q+i}\left(\mathcal{X}_{T_{i}}, i\right)$

$$
Z_{i}=\psi_{1,1}^{i}\left(\left[V \times T_{i}\right], f_{1}, \ldots, f_{i}\right) .
$$

I show by increasing induction on $i$ that $\overline{\operatorname{reg}}_{q+i, i}\left(Z_{i}\right) \neq 0$. Case $i=p-q$ implies Theorem 3

Case $i=0$ a straightforward adaptation of V2], Proposition 4: for $d \gg 0$ and for any open subset $U_{0} \subset T_{0}$ the cohomology class of the cycle $\left[V \times U_{0}\right] \in \mathrm{CH}^{q}\left(\mathcal{X}_{U_{0}}\right)$ in $\mathrm{H}^{2 q}\left(\mathcal{X}_{U_{0}}, \mathbb{C}\right)$ does not belong to $j_{U_{0}}^{*}\left(\mathrm{H}^{2 q}\left(\mathcal{Y}_{U_{0}}, \mathbb{C}\right)\right)$. 
Assume $i>0$. Then $T_{i-1} \cup T_{i}=P_{i} \backslash\left(P_{i} \cap Q_{i-1}\right)$, hence $T_{i-1}$ is a hyperplane section of $T_{i-1} \cup T_{i}$ and $T_{i}$ is the complementary open set. Hence there is a commutative diagram

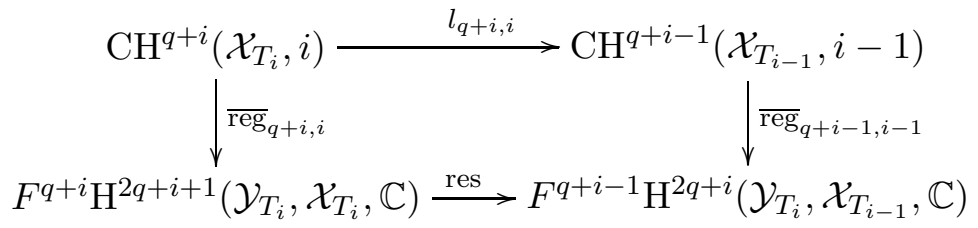

where res is the residue map and $l_{q+i, i}$ is the linking map for the long exact sequence of higher Chow groups. One has

$$
l_{q+i, i}\left(Z_{i}\right)=\left(l_{q, 0} \circ \psi_{1,1}^{i}\right)\left(\left[V \times T_{i}\right], f_{1}, \ldots, f_{i}\right)=\psi_{1,1}^{i-1}\left(\left[V \times T_{i-1}\right], f_{1}, \ldots, f_{i-1}\right)=Z_{i-1} .
$$

The induction assumption $\overline{\mathrm{reg}}_{q+i-1, i-1}\left(Z_{i-1}\right) \neq 0$, and the commutativity of the diagram implies $\overline{\operatorname{reg}}_{q+i, i}\left(Z_{i}\right) \neq 0$.

\section{Proof of Theorem 4}

4.1. Construction of the cycle $Z_{U}$. Let $P \subset Y$ be a codimension $q$ linear subspace, let $R \subset Y$ be a codimension $q+2$ linear subspace such that $R \subset P$ and let $S$ be the blow-up of $P$ along $R$. There are natural maps $\psi: S \rightarrow \mathbb{P}_{\mathbb{C}}^{1}$ (where $\mathbb{P}_{\mathbb{C}}^{1}$ parametrizes hyperplanes of $P$ containing $R$ ) and $s: S \rightarrow Y$. For any $x \in \mathbb{P}_{\mathbb{C}}^{1}$ let $L_{x}=s\left(\psi^{-1}(x)\right)$ denote the codimension $q+1$ linear subspace of $Y$. For any subvariety $B \subset \mathbb{P H}^{0}(Y, \mathcal{O}(d))$ let $C_{B}=S \times_{Y} \mathcal{X}_{B}$ and let $\psi_{B}: C_{B} \rightarrow \mathbb{P}_{\mathbb{C}}^{1}$ and $s_{B}: C_{B} \rightarrow \mathcal{X}_{B}$ be the morphisms deduced from $\psi$ and $s$ by base change.

Let $U \subset \mathbb{P} H^{0}(Y, \mathcal{O}(d))$ be the locus of hypersurfaces $X_{t}$ such that $\left[L_{0} \cap X_{t}\right]=d R$ and $\left[L_{\infty} \cap X_{t}\right]=d R$. One has $\operatorname{codim}\left(U, \mathbb{P H}^{0}(Y, \mathcal{O}(d))\right) \sim_{d \rightarrow \infty} 2 \frac{d^{N-q}}{(N-q) !}$. By construction, one has $s_{U}\left(\psi_{U}^{-1}(0)\right)=R \times U=s_{U}\left(\psi_{U}^{-1}(\infty)\right)$, hence the couple $\left(C_{U}, \psi_{U}\right)$ defines an element $Z_{U} \in \mathrm{CH}^{q+1}(U, 1)$.

4.2. Proof of $\overline{\operatorname{reg}}_{q+1,1}\left(Z_{U}\right) \neq 0$. Let $T \subset \mathbb{P H}^{0}(Y, \mathcal{O}(d))$ be the locus of hypersurfaces $X_{t}$ such that $L_{0} \subset X_{t}$ and $\left[L_{\infty} \cap X_{t}\right]=d R$. Then $T \subset T \cup U$ is a hyperplane section and $U \subset T \cup U$ is the complementary open set. Hence there is a natural linking homomorphism $l_{q+1,1}: \mathrm{CH}^{q+1}\left(\mathcal{X}_{U}, 1\right) \rightarrow \mathrm{CH}^{q}\left(\mathcal{X}_{T}\right)$. Since $C_{T}$ is the union of a variety dominating $\mathbb{P}_{\mathbb{C}}^{1}$ and of the variety $\psi_{T}^{-1}(0) \simeq L_{0} \times T$, one has $l_{q+1,1}\left(Z_{U}\right)=d\left[s_{T}\left(\psi_{T}^{-1}(0)\right)\right]=d\left[L_{0} \times T\right]$.

I now proceed as in section [3. A straightforward adaptation of the argument of $\mathrm{V2}$, Proposition 3 shows that for $d \gg 0$ the class $\mathrm{cl}_{q}\left(d\left[L_{0} \times T\right]\right) \in \mathrm{H}^{2 q}\left(\mathcal{X}_{T}, \mathbb{C}\right)$ does not belong to $j_{T}^{*}\left(\mathrm{H}^{2 q}\left(\mathcal{Y}_{T}, \mathbb{C}\right)\right.$, hence $\overline{\mathrm{cl}}_{q}\left(d\left[L_{0} \times T\right]\right) \in \mathrm{H}^{2 q+1}\left(\mathcal{Y}_{T}, \mathcal{X}_{T}, \mathbb{C}\right)$ is non-zero. Since the diagram

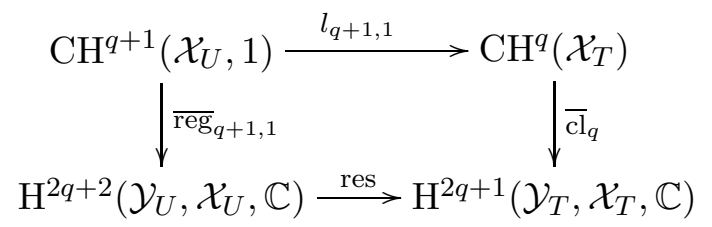

is commutative, it follows that $\overline{\operatorname{reg}}_{q+1,1}\left(Z_{U}\right) \neq 0$. 
4.3. Indecomposability of the cycle $Z_{U}$. Since $\overline{\mathrm{reg}}_{q+1,1}\left(Z_{U}\right) \neq 0$, it is enough to show that the space of decomposable cycles is contained in the kernel of $\overline{\mathrm{reg}}_{q+1,1}$.

Let $i \in\{1, \ldots, q\}, Z_{U}^{\prime} \in \mathrm{CH}^{i}\left(\mathcal{X}_{U}\right)$ and $Z_{U}^{\prime \prime} \in \mathrm{CH}^{q-i}\left(\mathcal{X}_{U}, 1\right)$. One has to show $\operatorname{reg}_{q+1,1}\left(\phi_{i, 0}\left(Z_{U}^{\prime} \otimes Z_{U}^{\prime \prime}\right)\right) \in j_{U}^{*} F^{q+1} \mathrm{H}^{2 q+1}\left(\mathcal{Y}_{U}, \mathbb{C}\right)$. Since the diagram

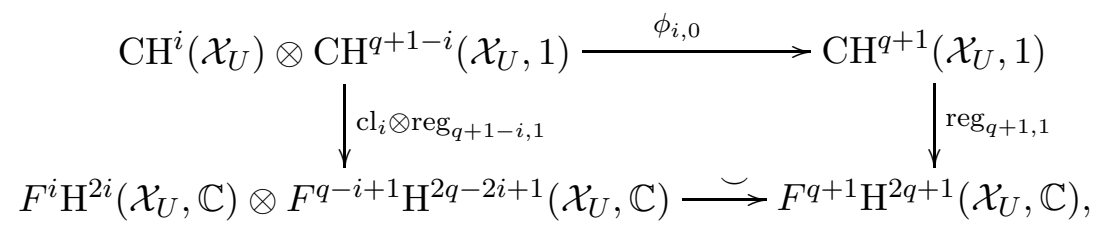

is commutative, it is enough to $\operatorname{show}_{i}\left(Z_{U}^{\prime}\right) \in \mathrm{H}^{2 i}\left(\mathcal{Y}_{U}, \mathbb{C}\right)$ and $\operatorname{reg}_{q+1-i, 1}\left(Z_{U}^{\prime \prime}\right) \in \mathrm{H}^{2 q-2 i+1}\left(\mathcal{Y}_{U}, \mathbb{C}\right)$.

If $i<q$ this follows from the isomorphisms given by Theorem [1]

$$
\begin{aligned}
F^{i} \mathrm{H}^{2 i}\left(\mathcal{X}_{U}, \mathbb{C}\right) & \simeq F^{i} \mathrm{H}^{2 i}\left(\mathcal{Y}_{U}, \mathbb{C}\right), \\
F^{q-i+1} \mathrm{H}^{2 q-2 i+1}\left(\mathcal{X}_{U}, \mathbb{C}\right) & \simeq F^{q-i+1} \mathrm{H}^{2 q-2 i+1}\left(\mathcal{Y}_{U}, \mathbb{C}\right)
\end{aligned}
$$

If $i=q$, one has

$$
\mathrm{CH}^{1}\left(\mathcal{X}_{U}, 1\right) \simeq \mathrm{H}^{0}\left(U, \mathcal{O}^{*}(U)\right) \simeq \mathrm{CH}^{1}\left(\mathcal{Y}_{U}, 1\right),
$$

hence $\operatorname{reg}_{1,1}\left(Z_{U}^{\prime \prime}\right) \in \mathrm{H}^{1}\left(\mathcal{Y}_{U}, \mathbb{C}\right)$; on the other hand, since $\mathcal{X}_{U}$ does not contain any flat family of cycles of codimension $q$ and degree less than two, by Theorem 2 one has $F^{q} \mathrm{H}^{2 q}\left(\mathcal{X}_{U}, \mathbb{C}\right) \simeq F^{q} \mathrm{H}^{2 q}\left(\mathcal{Y}_{U}, \mathbb{C}\right)$, hence $\operatorname{cl}_{q}\left(Z_{U}^{\prime}\right) \in \mathrm{H}^{2 q}\left(\mathcal{Y}_{U}, \mathbb{C}\right)$.

4.4. Indecomposability of the restriction of $Z_{U}$ to a very general fiber $\mathcal{X}_{u}, u \in U$. This follows from the indecomposability of $Z_{U}$ by Proposition 5 of $[\mathrm{V} 2$.

Aknowledgments. I would like to thank Stefan Müller-Stach for explaining to me what a higher Chow group is, and for a useful remark on conjecture 1

\section{REFERENCES}

[B] S. Bloch Algebraic cycles and higher K-theory. Adv. in Math. 61, no. 3, p. 267-304. (1986).

[G1] M. GREen. Restrictions of linear series to hyperplanes, and some results of Macaulay and Gotzmann. Algebraic curves and projective geometry, p. 76-86, LNM 1389, (1989).

[G2] M. Green. Koszul cohomology and geometry. Lectures on Riemann surfaces (Trieste, 1987), p. 177-200, Algebraic Curves and Projective Geometry (E. Ballico and C. Ciliberto, eds), p. 77-88, Springer LNM 1389, Berlin (1989).

[N] J. NAGEL. Effective bounds for Hodge-theoretic connectivity. J. Alg. Geom. 11,no 1, p. 1-32, (2002).

[No] M. NorI. Algebraic cycles and Hodge theoretic connectivity. Invent. math. 111 (2), p. 349-373 (1993).

[O] A. Otwinowska Variétés de Hodge. Submitted, (2001).

[V2] C. Voisin. Nori's connectivity theorem and Higher Chow groups. J. Inst. Mat. Jussieu, no 2, p. 307-329 (2000). 